\title{
SNS BEAM-IN-GAP CLEANING AND COLLIMATION
}

\author{
S. Cousineau, Indiana University, Bloomington, IN, USA \\ D. Davino, Brookhaven National Laboratory Upton, NY, USA \\ N. Catalan-Lasheras, CERN, Geneva, Switzerland \\ J. Holmes Oak Ridge National Laboratory, Oak Ridge, TN, USA
}

\begin{abstract}
The Spallation Neutron Source (SNS) under construction at Oak Ridge National Laboratory will operate with very high beam power and very strict beam loss requirements. A two stage collimation system will be used in the accumulator ring to localize beam loss and keep residual radiation to a level that will allow for hands-on maintenance of the machine. The collimators will also be used in conjunction with a set of fast kickers to remove the beam from the gap before the rise of the extraction kickers. We have installed a full-scale collimation package into the ORBIT particle-in-cell tracking code, and have used it optimize both the collimation system and the beam-in-gap cleaning process. Results of the studies are presented here.
\end{abstract}

\section{INTRODUCTION}

The Spallation Neutron Source currently under construction at Oak Ridge National Laboratory will deliver $1.4 \times 10^{14}$ to a liquid mercury target at a frequency of $60 \mathrm{~Hz}$ to create the most intense source of neutrons in the world. Due to the very high beam power, unprecedented control of beam loss will be required in order to minimize radiation activation of the machine and allow for hands-onmaintenance in most areas. In the accumulator ring specifically, uncontrolled beam losses are not to exceed $1 \times 10^{-4}$ of the total beam intensity, or $1 \mathrm{~W} / \mathrm{m}$ around the ring. Loss levels of this order can not be achieved without the use of collimators that capture high emittance particles before they intercept the machine aperture. For this purpose, the SNS ring will employ a sophisticated two-stage collimation system composed of four adjustable primary scrapers and four fixed-aperture secondary absorbers. The system is designed to absorb either $1 \times 10^{-3}$ of the beam intensity during every accumulation cycle, or two full beam pulses in the event of a failure scenario.

In addition to scraping the beam halo, the collimation system, together with a set of fast stripline kickers, will provide a means of cleaning the beam in the gap before the rise of the extraction kickers. The beam gap is expected to reach the $1 \times 10^{-4}$ intensity level during accumulation due to effects such as nuclear scattering and energy loss at the foil, RF noise, and collimation inefficiency. Loss of these particles during the rise of the extraction kicker fields translates into over $100 \mathrm{~W}$ of power deposition on the extraction region, far in excess of the $1 \mathrm{~W} / \mathrm{m}$ safety level. The proposed solution is to use the stripline kickers to excite the beam in the gap on the betatron resonance and deposit it into the collimation system before the rise of the kickers.

To optimize both the beam in gap (BIG) cleaning process and the collimation system in general, we have installed a complete collimation package into the particlein-cell tracking code, ORBIT [2]. The structure of the collimation routine is modeled after a standalone collimation code K2 [3], with routines adapted specifically for the SNS energy range. Physical processes included are multiple coulomb scattering, ionization energy loss, nuclear inelastic absorption and nuclear elastic scattering, rutherford scattering, and a detailed treatment of the collimator edge. The ORBIT framework provides a realistic accelerator environment and a complete suite of diagnostics that, combined with the collimation package, allow for detailed studies of how the beam interacts with the collimation system. We have used this program to optimize several parameters of the collimation system, to determine uncontrolled loss distributions around the ring, and to test the full beam in gap cleaning process. We dedicate the first half of this paper to optimization work associated solely with the collimation system, and the balance to the beam in gap cleaning simulations.

\section{COLLIMATION SYSTEM OPTIMIZATION}

Much of the design work for the collimators was completed before installation of the ORBIT collimation package, and detailed descriptions of the system have been published [3]. The goal of our work was not to redesign the system but to fine-tune such parameters as primary scraper thickness and material, aperture shape, and collimator location. A few results are discussed here.

In a two-stage collimation system, particles first intercept the primary collimators (scrapers) where they are imparted a momentum kick. For betatron collimation, the momentum must be translated into displacement before absorption by the secondary collimators can occur. An analytic expression exists for the optimum phase advance between the primary collimator that induces the kick and the secondary collimator that performs the absorption:

$$
\cos (\Delta \mu)=\sqrt{\left(\epsilon_{1} / \epsilon_{2}\right)}
$$

Where $\Delta \mu$ is the phase advance, and $\epsilon_{1}$ and $\epsilon_{2}$ are the emittance apertures of the primary and secondary collimators, respectively. This phase advance provides the cleanest absorption of the scraped beam by the secondary collimators. However, the equation neglects the existence of 
other limiting machine apertures. The multiple-coulombscattered beam is gaussian in nature and possesses a long tail that risks interaction with limiting ring apertures before optimum phase advance is reached. In the case of the phase-optimized SNS system, the scraped beam must negotiate two quadrupole doublets and over twelve meters of vacuum pipe in its passage from the primary scraper to the last of the secondary absorbers. Collimation simulations which include both beam pipe and quadrupole apertures show that about $7-8 \%$ of the scraped beam is lost in the vicinity of the second quadrupole doublet instead of the intended collimator. This motivates a change in location of the last collimator from after the quadrupole doublet to just before the doublet. The new arrangement represents a compromise between optimum phase advance and limiting geometric aperture. Simulations with the new layout result in almost no losses on the doublet, and an overall efficiency increase of about $7 \%$. The separation of the last collimator from its optimum phase advance does allow a portion of the scraped beam to escape the collimation system, but it is below the limiting ring aperture therefore completes the turn without interaction and is collimated on a subsequent pass through the system.

The role of the primary collimators, or the "scrapers", is to kick the halo particles into large emittances without significantly changing the beam energy. The SNS primary collimator is comprised of four tantalum scrapers mounted on copper supports at angles of $0,45,90$, and -45 degrees. Tantalum is chosen for its high melting point and good durability. The thickness of the scraper determines the impact parameters of particles on the secondaries, which in turn influences the absorption efficiency of these collimators. Figure 1 shows the dependence of the individual collimator efficiencies on the scraper thickness, where we invoke the definition efficiency $\equiv \frac{\# \text { absorbed }}{\# \text { impacted }}$, and normalize to the maximum efficiency (about 90\%).

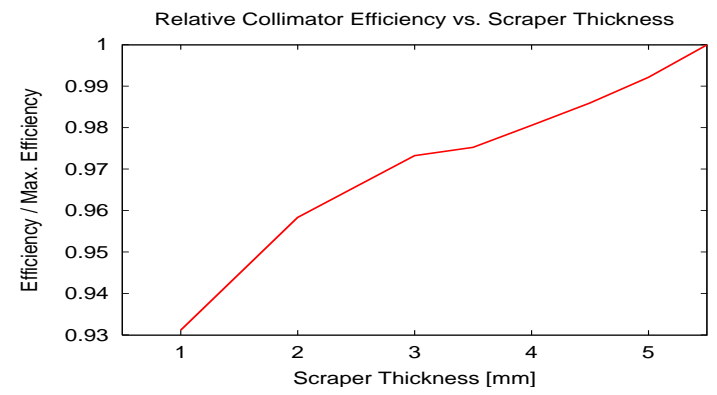

Figure 1: Individual collimator efficiency versus scraper thickness.

It is clear that the thicker scrapers provide better absorption efficiency. However, too thick of a scraper leads to scraped beam intercepting the quadrupole and beam pipe apertures before reaching the collimators. Based on our studies, the optimum scraper thickness for the SNS system appears to be $\approx 4.0 \mathrm{~mm}$.

Figure 2 demonstrates the effect of the fully optimized collimation system. The scrapers aperture was set to $200 \pi \mathrm{mm} \cdot \mathrm{mrad}$, and about $15 \%$ of the initial gaussian beam distribution exceeded that value. The initial emittance profile of the beam is plotted along with emittance profiles after 10 turns and after 100 turns. After 10 turns around the machine, the beam halo is almost completely absorbed. Since betatron phasing ensures that at least few turns will be needed for all of the halo to intercept the scrapers, a 10 turn cleaning success implies almost singleturn collimation once particle intercepts the system. Typical loss distributions around the ring show $88-90 \%$ of particles being absorbed directly in the collimators, $3-4 \%$ impacting the radiation-hard quadrupoles, and about $7 \%$ impacting the vacuum pipe. Overall, $98 \%$ of all losses occur within the collimation straight section. In our simulations, both the beam pipe and the quadrupoles are treated as black absorbers, whereas in reality some fraction of particles landing there will scatter into downstream collimators. Also, it should be noted that the loss distribution depends heavily on the vacuum pipe radius which is still under design; a conservative estimate of $12.0 \mathrm{~cm}$ radius was used for the simulation.

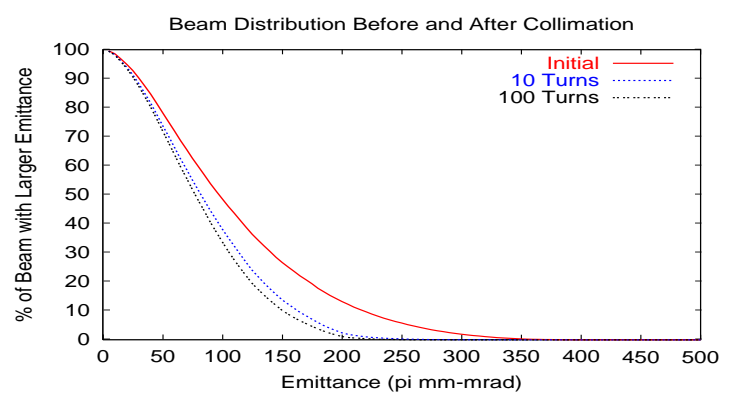

Figure 2: Beam emittance profiles before collimation (solid red line), after 10 turns (dashed blue line), and after 100 turns (dashed black line).

\section{BEAM IN GAP CLEANING}

Unlike the halo beam, gap particles do not necessarily have large transverse emittances and therefore will not reach the collimators without some type of intervention. Five vertical stripline kickers with a cumulative kicking strength of $1 \mathrm{mrad}$ will be used to drive the gap beam into the collimation system. The kickers are capable of reversing polarity on a turn-by-turn basis according to a programmable polarity sequence, and therefore they can be made fire in resonance with the vertical betatron tune of the ring. To determine the exact sequence of kicks for any given tune, an initial kick of arbitrary polarity is imparted and the beam centroid is followed around the phase space for subsequent turns. On each turn kicks are assigned with the same polarity as the centroid momentum. Though the effect of a kick on each individual particle is sensitive to the particle's phase, using the centroid in this manner guarantees that the net effect is always to increase the magnitude 
of the momentum in the distribution.

For very small tune spreads, it is only necessary for a sequence of kicks to follow the main tune of the lattice. However, the SNS expects tune spreads on the order of $0.15-0.2$. Kicking on resonance with the bare tune alone will serve to excite only a small fraction of the beam in the gap. We account for the range of tunes by defining entire kicking sequences for several tunes within the spread and apply them sequentially to excite the entire beam.

The simulations included initial gaussian beam distributions of finite momentum spread. The five kickers were modeled with a single, 1 mrad kick, with kick polarity sequences defined by the aforementioned method. Second order transport matrices were used to induce chromatic tune spread in the beam. The full baseline collimation system was used, with the variable-aperture primary scrapers set to $200 \pi \mathrm{mm} \cdot \mathrm{mrad}$. For SNS, the anticipated fractional momentum spread for the nominal (non-gap) beam is $\Delta p / p=0.007$, the RF acceptance is $\Delta p / p=0.010$, and the momentum aperture of the ring is $\Delta p / p=0.020$. Results of simulations with the three different initial beam momentum spreads are shown in Figure 3, where the fraction of particles lost is plotted over several turns of the beam. For each of the three cases, the entire beam is absorbed in under sixty turns around the ring.



Figure 3: Fraction of particles lost versus turn for three different momentum spreads: the solid red line is for $\Delta p / p=$ 0.007 , the dashed blue line is for $\Delta p / p=0.010$, and the dashed black line is for $\Delta p / p=0.020$.

The efficiency of the beam in gap cleaning process is fundamentally limited by the collimation efficiency. Gap particles that arrive at the primary collimator before any other aperture will be collimated in the same manner as the non-gap beam. However, the drift speed of the beam in the gap is much greater than the anticipated non-gap halo drift, and therefore it is possible for particles of particular phases to bypass the primary collimators altogether and impact secondary collimators instead. In our simulations, about $75 \%$ of the gap particles initially impact the primary scraper, while the remaining $25 \%$ overshoot into the secondaries; no particles initially impacted any of the other limiting apertures. Particles that pass directly to the secondary collimators are less likely to be absorbed than those that pass through the primary collimator first. Figure 4 shows typical primary impact parameters on both the scrapers and on one of the secondaries. The primary impact parameters on the secondaries are large, but not as large as they would have been had the particles been scattered by the primary scrapers. This leads to an overall decrease collimation efficiency for beam in gap particles compared to non-gap particles.
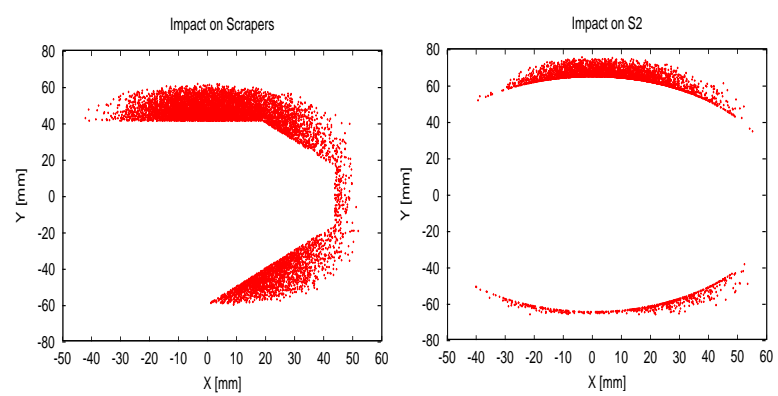

Figure 4: The figure on the left shows primary impact parameters of the beam in the gap on the four primary scrapers, and the figure on the right shows the primary impact parameters one of the secondary absorbers.

The two parameters that control the distribution of impacts among the collimators are the primary collimator aperture and the kicker strength. The primary aperture is fixed by the non-gap beam distribution and can not be varied to accomodate the beam in the gap. The other variable is kicker strength, which determines the drift velocity of the gap beam. A decrease in kicker strength would correspond to a slower, more controlled halo drift; however, this could also lead to considerably slower beam in gap cleaning.

\section{CONCLUSIONS}

A new collimation simulation package embedded in a particle-in-cell environment has allowed us to perform detailed studies of the SNS collimation system and beam-ingap cleaning process. Results of the work have lead to optimization of collimator location and scraper thickness. Additionally, we have shown that the collimation system can be used with a set of fast kickers to clean the beam in the gap before the rise of the extraction kickers.

This work is supported by SNS through UT-Battelle, LLC, under contract DE-AC05-00OR22725 for the U.S. Department of Energy. SNS is a partnership of six national laboratories: Argonne, Brookhaven, Jefferson, Lawrence Berkeley, Los Alamos, and Oak Ridge.

\section{REFERENCES}

[1] See K2 code description in N. Catalan-Lasheras, "Transverse and Longitudinal Beam Colliamtion in a High-Energy Proton Collider" PhD Thesis, University of Zaragoza, Spain (1999).

[2] J. Galambos et al., ORBIT User's Manual, SNS/ORNL/AP Technical Note 011, 1999.

[3] N. Catalan-Lasheras et. al., "Optimization of the collimation system for the Spallation Neutron Source accumulator ring”, Phys. Rev. ST Accel. Beams 4, 010101 (2001)). 